\title{
-2proPSA and Prostate Health Index Usefulness for the Diagnosis of Prostate Cancer for PSA Range between 3 and $10 \mathrm{ng} / \mathrm{ml}$
}

\section{J Juan Escudero, A Duran-Rivera*, E Escudero, A Montoro, M Fabuel del Toro, M Ramos de Campos and E López Alcina}

University General Hospital of Valencia, Spain

*Corresponding author: Andrea Durán-Rivera, University General Hospital of Valencia, Tres Cruces \#2, 46014, Valencia, Spain, Tel: 6220-581580,E-mail: andreajduran@gmail.com

\begin{abstract}
Introduction: The high incidence of prostate cancer (PCa) worldwide and the growing interest in overdiagnosis and overtreatment make the study of new markers imperative for helping us predict the presence and aggressiveness of $\mathrm{PCa}$. The objective of this work is to evaluate the -2proPSA and Prostate Health Index Usefulness for the diagnosis of PCa.
\end{abstract}

Material and methods: A prospective study including 101 patients with PSA levels between $3-10 \mathrm{ng} / \mathrm{mL}$ and normal digital rectal exam (DRE) was conducted between November 2013 and November 2014. All patients underwent prostate biopsy and PSA, free PSA and -2proPSA determination. -2proPSA ratio (\%2proPSA) and Prostate Health Index (PHI) were also calculated.

Results: Patients had a mean age of 63.7 years old. The means of PSA and free PSA ratio (\%fPSA) were $6.06 \mathrm{ng} / \mathrm{mL}$ and $16 \%$, respectively. The means of -2proPSA and \%2proPSA were $16.8 \%$ and $1.8 \%$, respectively. Prostate volume mean was $46 \mathrm{cc}$ and PSA density mean were $0.19 \mathrm{ng} / \mathrm{cc}$.

In the univariate analysis, only \%fPSA and PHI showed statistical significant association with the presence of tumor in prostate biopsy, whereas \%2proPSA almost reached statistical significance.

In the multivariate analysis, $\mathrm{PHI}$ showed the best area under the curve (AUC) with a value of 0.749 , followed by $\%$ fPSA (0.708) and -2proPSA (0.671).

The best values for internal and external validity of each of the evaluated parameters turned out to be for $\mathrm{PHI}$, with $93 \%$ sensibility and $37 \%$ specificity, $53 \%$ positive predictive value (PPV) and $88 \%$ negative predictive value (NPV).

Conclusions: $\mathrm{PHI}$ is the parameter that allows predicting the presence of PCa more precisely for patients with normal (DRE) and PSA between 3 and $10 \mathrm{ng} / \mathrm{mL}$.

\section{Introduction}

Prostate cancer (PCa) is the most frequent neoplasia among adult men, although its elevated incidence does not translate in a higher mortality rate.

The prostate specific antigen (PSA) is a kallikrein protein family member and since the 1990s has been used as a biochemical marker for the early diagnosis of PCa. Since the introduction of PSA, we have witnessed the migration of this tumor to initial stages diagnosis, with a subsequent decrease in mortality. Even though PSA use is constantly challenged due to its low specificity, especially in what is known as the PSA grey area (between 4-10 ng/dL range), PSA is still the most utilized tumor marker for PCa [1].

In this sense, aiming to improve this parameter, new biomarkers have been studied showing varying results. PSA by-products, such as free PSA (fPSA), \%fPSA ( $F P S A / t o t a l$ PSA) or PSA density (PSAD), and transitional zone PSA density have shown a limited benefit when it comes to improving PSA specificity. In this manner, between 60 to $70 \%$ of patients who undergo prostate biopsy, which was indicated based on this parameters only, end up having a negative result.

PSA is almost exclusively produced by the prostate acinar epithelium. The proPSA is an inactive precursor composed of 244 amino acids and is also secreted by the prostatic cells. There are four different isoforms of proPSA and includes integrated and truncated variants. ProPSA seems to be a more cancer specific structure of PSA, due to the fact that is specially expressed in the peripheral zone, while undetectable in the transition zone.

Citation: Escudero JJ, Duran-Rivera A, Escudero E, Montoro A, del Toro MF, et al. (2018) -2proPSA and Prostate Health Index Usefulness for the Diagnosis of Prostate Cancer for PSA Range between 3 and 10 ng/ml. Int Arch Urol Complic 4:041. doi.org/10.23937/2469-5742/1510041

Accepted: Augsut 06, 2018: Published: Augsut 08, 2018

Copyright: (c) 2018 Escudero JJ, et al. This is an open-access article distributed under the terms of the Creative Commons Attribution License, which permits unrestricted use, distribution, and reproduction in any medium, provided the original author and source are credited. 
The -2proPSA is a free PSA truncated variant and is also the more predominant proPSA isoform isolated from prostate cancer tissues. In patients without tumor, it represents $6-20 \%$ of the total circulating free PSA [2]. However, it may reach $25-95 \%$ in patients with PCa.

Prostate Health Index (PHI) is a formula that combines Total PSA, free PSA and -2proPSA ([-2]proPSA/free $P S A) \times V P S A$ ) in one score, aiming to improve cancer detection and decision making.

The objective of this work is to evaluate the usefulness of -2proPSA and PHI for the diagnosis of prostate cancer.

\section{Material and Methods}

After approval of the hospital Ethics Committee, a prospective study was designed. All patients who underwent prostatic biopsy, fulfilling all the inclusion criteria and none of the exclusion criteria, were included in the study (Table 1 ).

Patients referred to our center for prostate biopsy, as well as those indicated for biopsy directly from our outpatient clinic, were included in the study between November 2013 and November 2014. After meeting the inclusion criteria, a total of 101 patients were evaluated.

Once patients were informed about the study and after obtaining oral and written informed consent, blood samples were collected for the analysis of PSA, free PSA, and -2proPSA determinations. Subsequently, all patients underwent echodirected transrectal prostate biopsy. Twelve cores were collected in a systematic fashion, directing the punctures to the more lateral and posterior aspects of the prostate gland. All biopsies were performed as an outpatient procedure and under local anesthesia. Prostate volume was also calculated with the transrectal ultrasound.

The variables included in this study are as follows: age, PSA, \%fPSA, prostatic volume, -2proPSA, \%2proPSA $[(-2$ proPSA/free PSA $\times 1000) \times 100]$ and Prostate Health Index (PHI). The biopsy result, interpreted as positive or

Table 1: Inclusion and exclusion criteria.

\section{Inclusion criteria}

PSA between 3 y $10 \mathrm{ng} / \mathrm{mL}$

Normal digital rectal exam

Informed consent

First prostatic biopsy

\section{Exclusion criteria}

Previous prostate biopsy

Previous prostatic surgery Prostatitis

Under treatment with $5 \mathrm{ARI}$ negative for tumor, was the independent variable. Intraepithelial neoplasia and atypical small acinar proliferation were interpreted as negative for tumor.

All prostate biopsies were guided with transrectal ultrasound. Following the same pattern, twelve cores were obtained, six per each prostatic lobe using $2 \%$ lidocaine as local anesthetic. A single urologist performed all biopsies. The samples were collected in color twelve individual containers and sent for analysis by two different uropathologist.

After evaluating the normal distribution of the variables with Kolmogorov-Smirnov test, a Student t-test was conducted for independent variables. In a second step, the area under the curve (AUC) was determined for the most relevant parameters. All data was processed and analyzed using the statistical package SPSS-21.

\section{Results}

For the period between November 2013 and November 2014, a total of 115 patients were selected to participate in the study. Finally, only 101 were included. 14 patients were excluded from the study due to the presence of lower urinary tract symptoms compatible with urinary infection or because of the lack of informed consent. Population characteristics subdivided by presence vs. absence of tumor are presented in Table 2.

From these 101 patients, 44 cases (43.6\%) had a prostatic tumor in the biopsy and 57 cases $(56.4 \%)$ had a normal biopsy. The mean age of the population was 63.7 years old. The following mean values were obtained: $6.06 \mathrm{ng} / \mathrm{ml}$ for PSA, $16 \%$ for \%fPSA, the -2proPSA mean was $16.8 \mathrm{ng} /$ $\mathrm{ml}, 1.8 \%$ for $\% 2 \mathrm{proPSA}$, the mean prostatic volume was 46 cc and $0.19 \mathrm{ng} / \mathrm{cc}$ for PSA density. The overview of the descriptive analysis of population characteristics, subdivided by the presence vs. absence of tumor, and the results of the univariate analysis are displayed in Table 2. PSA Ratio and $\mathrm{PHI}$ are the only parameters that demonstrated a statistically significant association with the presence of PCa.

Table 2 presents how -2proPSA, \%2proPSA and PHI showed superior central tendency measurements in the group of patients with prostate neoplasia. Nevertheless, this tendency is also exhibited by total PSA, \%fPSA and PSAD. It is worth noting that prostates with tumor had a smaller mean prostate volume compared with the non-tumoral group.

Table 2: Population characteristic subdivided by presence vs. absence of tumor. Univariate analysis.

\begin{tabular}{|l|l|l|l|l|}
\hline & Global & No tumoral & Tumoral & p \\
\hline Age & $63.79+/-7.5$ & $52.56+/-7.7$ & $65.39+/-6.9$ & 0.16 \\
\hline Prostate volume & $46.6+/-24.2$ & $51.51+/-26.4$ & $40.29+/-19.5$ & 0.56 \\
\hline PSAd & $0.19+/-0.19$ & $0.17+/-0.23$ & $0.21+/-0.14$ & 0.98 \\
\hline PSA & $6.06+/-1.9$ & $5.63+/-2.01$ & $6.6+/-1.7$ & 0.41 \\
\hline Free PSA & $1.01+/-0.61$ & $1.0+/-0.69$ & $0.9+/-0.47$ & 0.12 \\
\hline \%fPSA & $0.16+/-0.07$ & $0.18+/-0.07$ & $0.13+/-0.05$ & $\mathbf{0 . 0 4}$ \\
\hline -2proSPA & $16.88+/-11.15$ & $15.23+/-9.6$ & $19.03+/-12.6$ & 0.17 \\
\hline \%2proPSA & $1.81+/-1.08$ & $1.57+/-0.84$ & $2.1+/-1.26$ & 0.05 \\
\hline PHI & $44.02+/-26.04$ & $36.11+/-19.3$ & $54.27+/-29.9$ & $\mathbf{0 . 0 3}$ \\
\hline
\end{tabular}




\section{Curva COR}

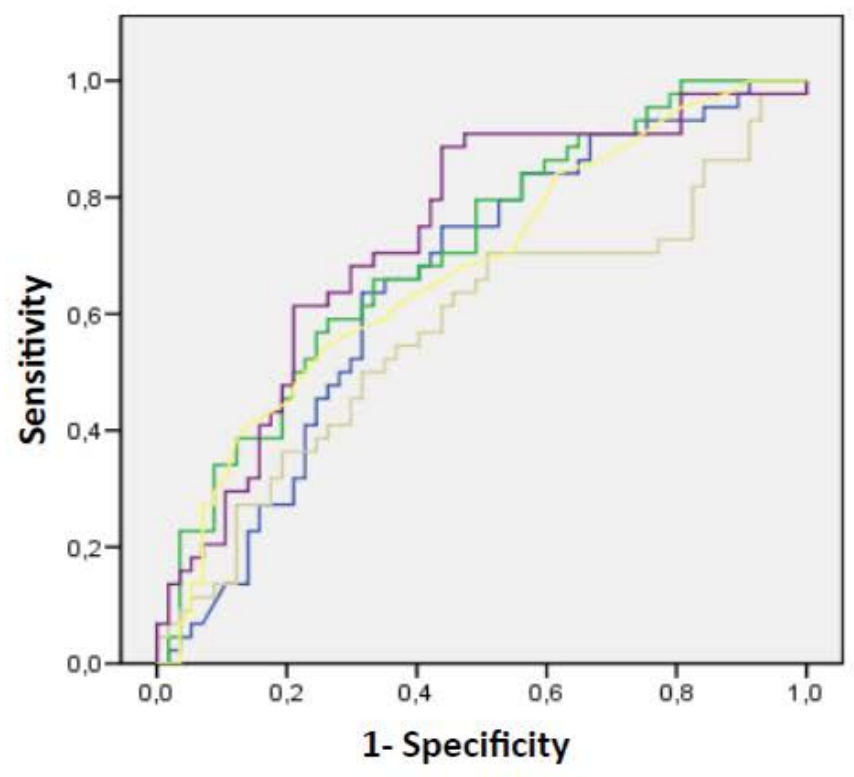




\section{Curva COR}
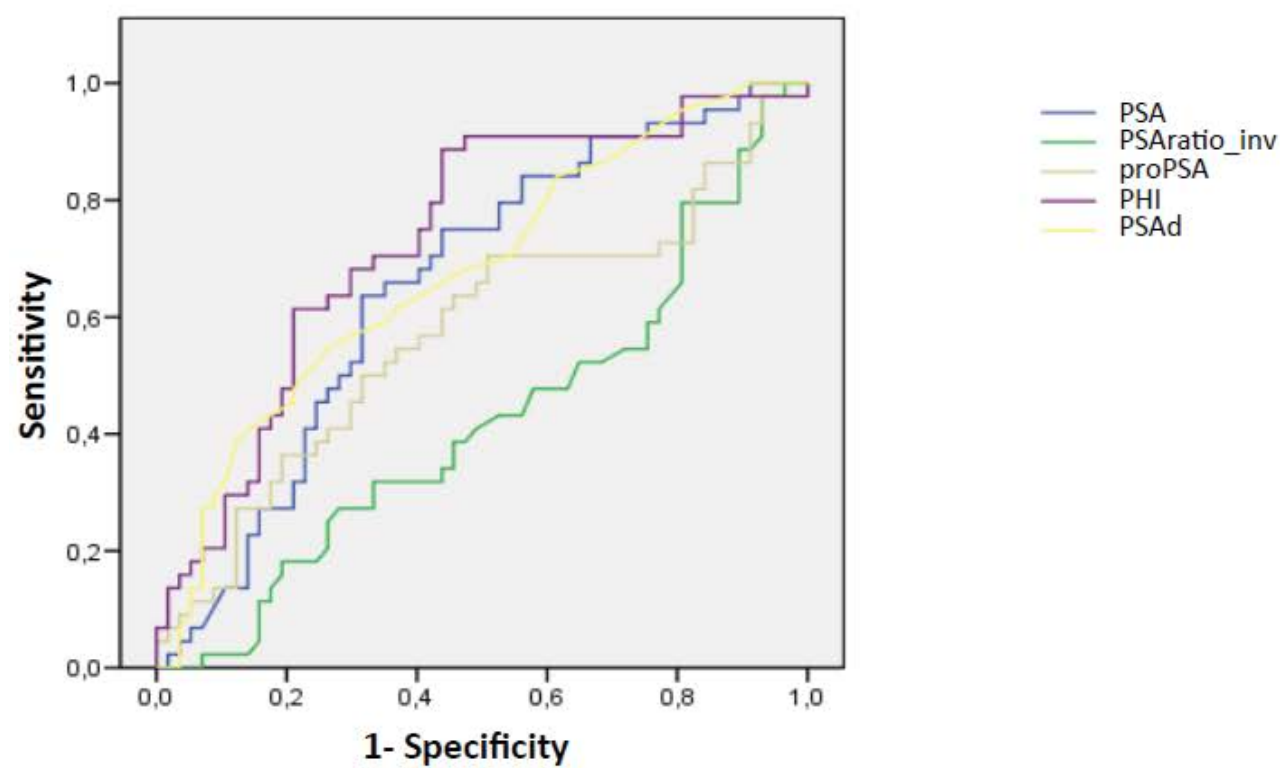

Figure 2: ROC curves of the most relevant parameters for diagnose of significant tumor.

to predicting the presence of $\mathrm{PCa}$, not only in the initial but also in subsequent prostate biopsies, increasing the specificity of total PSA and \%fPSA for the detection of PCa in long cohorts and multicenter studies [4].

In our center, after at least two PSA determinations under optimal conditions, prostate biopsy is indicated routinely in patients older than 65-years-old with PSA higher than $4 \mathrm{ng} / \mathrm{dl}$, and in patients either under 65-years-old or with any family history of PCa and with a PSA higher than $3 \mathrm{ng} / \mathrm{dl}$. Routinely, we do not take into consideration \%fPSA or PSAD for indication of the initial biopsy.

Some authors have reported the usefulness of -2proPSA and PHI determination on patients with lower PSA values, between $2 \mathrm{ng} / \mathrm{dl}$ and $4 \mathrm{ng} / \mathrm{dl}$ [5]. It has been described that, whereas PSA and \%fPSA remained comparable among the groups with and without tumor, $\% 2$ proPSA was higher in the group of patients with tumor and its specificity and AUC in the ROC curve were clearly superior. On the other hand, Catalona, et al. revealed that patients with low PSA but elevated \%2proPSA tend to be diagnosed with intermediate and high-risk tumors [6].

In this case, it was decided not to modify the cutoff points indicating the prostate biopsy, until the usefulness of the new marker was proven, which can be considered a task for future investigation. Other authors have already proposed the usefulness of -2 proPSA and its derivatives for the diagnosis of $\mathrm{PCa}$ in patients with PSA levels between 4 and $10 \mathrm{ng} / \mathrm{dl}[7,8]$ and how the risk of prostate cancer raises up to 8 times in cases where the -2proPSA is found in the superior quartile [9].

In this study, we present the results of a prospective cohort with a follow-up adherence close to $100 \%$.
Selected patients were all candidates for a first biopsy. Even though the limited number of cases may represent a limitation of the study, the patients' standardized selection and the scheme for the biopsy procedure, as well as the fact that all biopsies were performed by a single urologist, offers guarantee of homogeneity in the process that multicenter studies cannot provide.

In the univariate study, only $\mathrm{PHI}$ and \%fPSA seem to be able to predict the presence of PCa in the prostate biopsy, whereas neither -2proPSA not \%2proPSA reveled statistically significant differences, although \%2proPSA was fairly close to statistical significance. It is possible that with a larger number of patients, the results may have been more in accordance with results published in previous series [10].

With the purpose of avoiding selection bias, patients with an abnormal (suggestive of tumor) digital rectal exam were excluded. In this manner, our AUC was at the level of other published series with a similar study design, such as Catalona and Guazzoni [3,4]. Other authors have included in their series patients with tumor-suspicious digital rectal exams [2], describing an AUC of 0.74 , which is very similar to our findings. This fact encourages us to consider the use of $\mathrm{PHI}$ regarding the PSA results for future studies.

The PHI internal validity values were calculated from the quartiles obtained in our series. The cut-off point was defined by the value of the first quartile, positioning $\mathrm{PHI}$ at 28.2. Our results are slightly superior to other findings reported in larger population studies $[4,10,11]$ that had also used 28 as the cut-off values for PHI, however in this study no previous sensitivity value was set. It is also important to point out that using the first quartile of the results of this study as cut-off point, only three tu- 
mors were found in the inferior values. Moreover, none of them showed a Gleason score over six, and no more than three affected cores. They were all low-risk tumors.

The detection of aggressive tumors plus a reduction of unnecessary biopsies that would detect low-risk tumors is one of the hottest topics in urology nowadays. The usefulness of -2proPSA and PHI has been widely revealed in various studies. $\mathrm{PHI}$ increase has been correlated with the presence of tumors with Gleason score 7 or higher $[2,12,13]$ and also has served as the best parameter that allows differentiating the presence of aggressive tumors. Additionally, the expression of this phenomenon has also been studied in the radical prostatectomy samples, finding that $\mathrm{PHI}$ and \%proPSA may predict the presence of tumors with Gleason score more than 7, locally advanced tumors and tumors with a higher volume.

Even though it was not the primary purpose of this study, a sub-analysis was performed to evaluate the usefulness of the diagnosis of intermediate and highrisk tumors with Gleason score of 7 or higher. In this manner, the results were less clear. In the univariate analysis, the -2proPSA seems to be the parameter that best predicts the presence of a Gleason score of 7 or higher in the prostate biopsy sample. However, in the multivariate analysis, the parameter that presented the best AUC was PSA followed by PHI and -2proPSA, although the differences were almost imperceptible. According to these results, the data does not match those published in the literature $[2,14]$.

The suitability of -2proPSA and PHI as part of active surveillance protocols has been described previously [15]. Such role has not yet been evaluated in our series. A broader follow-up will help clarify this topic.

As described in some works, $\mathrm{PHI}$ application in daily practice may help spare 8 to $20 \%$ of prostate biopsies [4]. The economic impact of the use of this technique has not been deeply studied until now, some economic aspects have been published [16], they described that economic reduction is more important in the group of patients with low PSA (higher than $2 \mathrm{ng} / \mathrm{dl}$ ).

\section{Conclusions}

In the present study, $\mathrm{PHI}$ has demonstrated the best AUC for the detection of PCa in the first biopsy for patients with normal digital rectal exam and PSA between 3 and $10 \mathrm{ng} / \mathrm{dl}$. PHI has also shown a higher specificity than PSA and \%fPSA for a cut-off point of 28.

\section{References}

1. Catalona WJ, Partin AW, Slawin KM, Brawer MK, Flanigan RC, et al. (1998) Use of the percentage of free prostate-specific antigen to enhance differentiation of prostate cancer from benign prostatic disease: A prospective multicentre clinical trial. JAMA 279: 1542-1547.

2. Stephan C, Vincendeau S, Houlgatte A (2013) Multicenter evaluation of -2 prostate specific antigen and the prostate health index for detecting prostate cancer. Clinical Chemis- try 59: 306-314.

3. Guazzoni G, Nava L, Lazzeri M, Scattoni V, Lughezzani G, et al. (2011) Prostate-Specific Antigen (PSA) Isoform p2PSA significantly improves the prediction of prostate cancer at initial extended prostate biopsies in patients with total PSA between 2.0 and $10 \mathrm{ng} / \mathrm{mL}$ : Results of a prospective study in a clinical setting. Euro Urol 60: 214-222.

4. Catalona WJ, Partin AW, Sanda MG, Wei JT, Klee GG, et al. (2011) A multicenter study of [-2]pro prostate specific antigen combined with prostate specific antigen and free prostate specific antigen for prostate cancer detection in the 2.0 to $10.0 \mathrm{ng} / \mathrm{mL}$ prostate specific antigen range. J Urol 185: $1650-1655$.

5. Sokoll LJ, Chan DW, Mikolajezyk SD, Rittenhouse HG, Evans CL, et al. (2003) Proenzime PSA for the early detection of prostate cancer in the $2.5-4 \mathrm{ng} / \mathrm{mL}$ total PSA range: Preliminary analysis. Urology 61: 274-276.

6. Catalona WJ, Bartsch G, Rittenhouse HG, Evans CL, Linton $\mathrm{HJ}$, et al. (2004) Serum pro-prostate specific antigen preferentially detects aggressive prostate cancers in men with 2 to 4 $\mathrm{ng} / \mathrm{mL}$ prostate speciic antigen. J Urol 171: 2239-2244.

7. Stephan C, Stroebel G, Heinau M, Lenz A, Roemer A, et al. (2005) The ratio of prostate-specific antigen (PSA) to prostate volume (PSA density) as a parameter to improve the detection of prostate carcinoma in the range of $<4 \mathrm{ng} /$ $\mathrm{mL}$. Cancer 104: 993-1003.

8. Mikolajczyk SD, Catalona WJ, Evans CL, Linton HJ, Millar LS, et al. (2004) Proenizme forms of the prostate specific antigen in serum improve the detection of prostate cáncer. Clin Chem 50: 1017-1025.

9. Rhodes T, Jacobson DJ, McGree ME, St Sauver JL, Sarma AV, et al. (2012) Distribution and association of [-2]proenzyme-prostate specific antigen in community dwelling black and white men. J Urol 187: 92-96.

10. Jansen $\mathrm{FH}$, Van SChaik $\mathrm{RH}$, Kurstjens J, Horninger W, Klocker $\mathrm{H}$, et al. (2012) Prostate-specific antigen (PSA) isoform p2PSA in combination with total PSA and free PSA improves diagnostic accuracy in prostate cancer detection. Eur Urol 57: 921-927.

11. Le BV, Griffin CR, Leob S, Carvalhal GF, Kan D, et al. (2010) [-2]proenzime prostate specific antigen is more acurate than total and free prostate specific antigen in differentiating prostate cancer from benign disease in a prospective prostate cancer screening study. J Urol 183: 1335-1339.

12. Wang W, Wang M, Wang L, Adams TS, Tian Y, et al. (2014) Diagnostic ability of \%2p2PSA and prostate health index for aggressive prostate cancer: A meta-analysis. Sci Rep 4: 5012-5020.

13. Loeb S, Sanda MG, Broyles DL, Shin SS, Bangma CH, et al. (2015) The prostate health index selectively indentifies clinically significant prostate cancer. J Urol 193: 1163-1169.

14. Guazzoni G, Lazzeri M, Nava L, Lughezzani G, Larcher A, et al. (2012) Preoperative prostate-specific antigen isoform p2PSA and its derivates, \%2pPSA and prostate health index, predict pathologic outcomes in patients undergoing radical prostatectomy for prostate cancer. Euro Urol 61: 455-466.

15. Tosoian J, Loeb S, Feng Z, Isharwal S, Landis $P$, et al. (2012) Association of [-2]proPSA with biopsy reclassification during active surveillance for prostate cancer. $\mathrm{J}$ Urol 188: 1131-1136.

16. Nichol MB, Wu J, An JJ, Huang J, Denham D, et al. (2011) Budget impact analysis of a new prostate cancer risk index for prostate cancer detection. Prostate Cancer Prostatic Dis 14: $253-261$. 